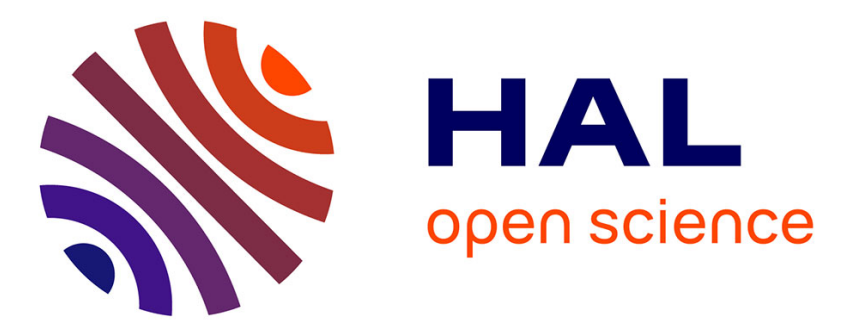

\title{
Towards Kinematics From Motion: Unknown Input Observer and Dynamic Extension Approach
}

Rayane Benyoucef, Hicham Hadj-Abdelkader, Lamri Nehaoua, Hichem Arioui

\section{To cite this version:}

Rayane Benyoucef, Hicham Hadj-Abdelkader, Lamri Nehaoua, Hichem Arioui. Towards Kinematics From Motion: Unknown Input Observer and Dynamic Extension Approach. IEEE Control Systems Letters, 2021, 6, pp.1340-1345. 10.1109/LCSYS.2021.3093067 . hal-03321836

\section{HAL Id: hal-03321836 https://hal.science/hal-03321836}

Submitted on 28 Dec 2021

HAL is a multi-disciplinary open access archive for the deposit and dissemination of scientific research documents, whether they are published or not. The documents may come from teaching and research institutions in France or abroad, or from public or private research centers.
L'archive ouverte pluridisciplinaire HAL, est destinée au dépôt et à la diffusion de documents scientifiques de niveau recherche, publiés ou non, émanant des établissements d'enseignement et de recherche français ou étrangers, des laboratoires publics ou privés. 


\title{
Towards Kinematics from Motion : Unknown Input Observer \& dynamic extension approach
}

\author{
R. Benyoucef, H. Hadj-Abdelkader, L. Nehaoua and H. Arioui
}

\begin{abstract}
This paper addresses an unknown input observer design to estimate simultaneously the 3D depth of a tracked image feature and the camera linear velocity using a low cost monocular camera and inertial sensor. The camera kinematic model is at first, augmented via the dynamic extension approach then described as a quasi-Linear Parameter Varying (qLPV) model. Further, the qLPV system is transformed into TakagiSugeno (T-S) form with unmeasured premise variables. The error convergence analysis is performed based on Lyapunov theory and Input to State Stability (ISS) property to ensure the boundedness of the state estimation error. Gains that guarantee the asymptotic stability of the estimation error can be properly computed by means of Linear Matrix Inequalities (LMIs). Finally the proposed approach is validated using synthetic data.
\end{abstract}

Keywords: Unknown Input Observer (UIO), Input to State Stability (ISS), qLPV Systems, Structure from Motion, LMI constraints, Lyapunov Theory

\section{INTRODUCTION}

Over the years, there has been significant interest in recovering the $3 \mathrm{D}$ structure of a scene from 2D pixels considering a sequence of images. Several solutions have been proposed in the literature for this issue. In robotics community, this problem can be referred to as Monocular Simultaneous Localization And Mapping (SLAM) and in computer vision community, it has been addressed as Structure from Motion (SfM) problem. Many researchers utilize the terms interchangeably [15] since both schemes aim at recovering the 3D structure of the scene.

Recent studies have developed a number of novel techniques to solve SfM problem using filtering schemes. For instance an Extended Kalman Filter (EKF) based approaches have been proposed in [1]-[3]. One can find in [4] and [5] the implementation of Unscented Kalman filter. Usually, the main drawback of filtering strategies is that they require a proper initialisation of the iterative process and they imply a certain degree of linearisation. Furthermore, many works have adopted deterministic nonlinear observers based approaches to recover the 3D structure of the tracked features. Among the first contribution one can cite work of Deluca and al. [6], where a nonlinear observer is designed to estimate the depth information with precise knowledge of the camera displacement.

It is thoroughly addressed in literature that the camera translational motion has a strong effect on the performance of 3D structure estimation [6], [7] and it is commonly known that estimating the depth is impossible if the camera is moving

All authors are with IBISC Lab, Univ. Evry, University of ParisSaclay University, 40 Rue du Pelvoux, 91080 Courcouronnes. France rayane.benyoucefduniv-evry. fr along the feature projection ray. In this context, particular attention has been devoted to active structure estimation from motion strategy where the camera trajectory is suitably chosen to optimize the convergence rate of the estimation error. A common methodology consists on imposing a desired transient response to the estimation error by acting on the camera linear velocity as addressed in [7]. This idea is extended to include other geometrical primitives such as lines [8], spherical and cylindrical targets [9] and moments [21]. Generally, when dealing with the SfM problem, a precise prior knowledge of the camera velocity is required to recover the 3D structure of the viewed scene. Measuring the camera velocity might be achieved if the camera is attached on an end effector of a robot manipulator fixed to the ground or by using dedicated sensors like Doppler effect radars. However, it is not a trivial task if the camera is mounted on a mobile or flying robot. In this respect, many works attempted to overcome the explicit dependence of the camera velocity on the estimation performance. For instance, in [10] and [11], a rotation-free active SfM algorithm scheme is designed considering some rotational invariant visual features obtained from spherical-projection model.

With respect to the existing literature and to the best to the authors knowledge, few works have addressed the problem of estimating the depth and the linear velocity simultaneously. One can cite [22], where a nonlinear observer is designed to estimate the depth, linear velocity, and the attitude based on horizontal plane assumption which means that this approach fails in case of inclined planes. Moreover, in [23], the authors developed a reduced order observer that only needs one camera linear velocity and corresponding acceleration to asymptotically estimate the depth information of the feature point attached to an object and the remaining camera velocities. Nevertheless, they assume a known uncertain dynamic model of the linear velocity.

The purpose of this work is to exploit images and Inertial Measurements, to eventually estimate the depth information of a fixed point feature in the scene and also to recover the linear velocity of the camera within its reference frame in order to overcome real time availability of the linear camera velocity. The nonlinear projection model of a perspective camera of a point feature is formulated as a qLPV system. The idea to immerse the given system dynamic into an augmented model [16] that has an appropriate structure to build an Unknown Input observer. The nonlinearities of the system under study are in function of the measured/unmeasured bounded states of the system. The stability analysis is discussed based on Lyapunov theory and ISS property. the LMIs constraints 
are established with minimized ISS gain based on the $H_{\infty}$ norm to ensure an explicit bound of the estimation error.

The remaining of this paper is organized as follows: Section II reviews the camera projection model and introduces basic definitions and notations. Section III deals with the qLPV representation of the vision system. The observer design is demonstrated in Section IV. Section V illustrates the performance of the proposed approach using synthetic image sequence. Finally, conclusions and remarks are given in Section VI

\section{BACKGROUND}

\section{A. Notations and basic definitions}

Throughout the paper, we adopt the following notations: matrices will be represented in upper case bold letters $\mathbf{X}$ and vectors in lower case bold letters $\mathbf{x} . \mathbf{X}=\mathbf{X}^{\top}$ refer to the symmetric matrix. (. $)^{-1}$ is the inverse of a given square matrix. $\mathbf{X}>0$ (resp. $\mathbf{X}<0)$ means that $\mathbf{X}$ is a definite positive (resp. negative) matrix. $\mathbf{0}$ and $\mathbf{I}$ refers to zero and identity matrices with appropriate dimensions. [.] $]_{\times}$ denotes the skew-symmetric matrix of a given vector and $\|()$.$\| represents the Euclidean norm. The infinity norm is$ designated by $\|(.)\|_{\infty}$.

In the subsequent, we recall some definitions to demonstrate the observer design procedure.

\section{Definition 1:}

- A scalar continuous function $\alpha(r)$, defined for $r \epsilon$ $[0, a)$ belongs to class $\mathcal{K}$ if it is strictly increasing and $\alpha(0)=0$.

- A scalar continuous function $\beta(r, s)$, defined for $r \epsilon$ $[0, a)$ and $s \in[0, \infty)$ belongs to class $\mathcal{K} \mathcal{L}$ if for each fixed s, the mapping $\beta(r, s)$ belongs to class $\mathcal{K}$ with respect to $r$ and for each fixed $r$, the mapping $\beta(r, s)$ is decreasing with respect to $s$ and $\beta(r, s) \rightarrow 0$ as $s \rightarrow \infty$.

Definition 2: Given a system:

$$
\dot{\mathbf{x}}(t)=\mathbf{f}(\mathbf{x}(t), \mathbf{u}(t))
$$

The system (1) is Input to State Stable if there exist a function $\beta_{\mathcal{K} \mathcal{L}} \in \mathcal{K} \mathcal{L}$ and $\beta_{\mathcal{K}} \in \mathcal{K}$ such that the trajectory associated for each initial condition $\mathbf{x}(0)$ and each input $\mathbf{u}(t)$ satisfying $\|\mathbf{u}(t)\|_{\infty}<\infty$ fulfills the following expression:

$$
\|(\mathbf{x}(t))\|<\beta_{\mathcal{K} \mathcal{L}}(\|(\mathbf{x}(0))\|)+\beta_{\mathcal{K}}\left(\|(\mathbf{u}(t))\|_{\infty}\right)
$$

Lemma 1: For every matrix $\mathbf{G}=\mathbf{G}^{T}>0, \mathbf{X}$ and $\mathbf{Y}$ with appropriate dimensions, the property below is satisfied:

$$
\mathbf{X}^{T} \mathbf{Y}+\mathbf{Y}^{T} \mathbf{X} \leq \mathbf{X}^{T} \mathbf{G X}+\mathbf{Y}^{T} \mathbf{G}^{-1} \mathbf{Y}
$$

Lemma 2 (Schur complement lemma): Consider the following convex nonlinear inequalities:

$$
\mathbf{R}>0, \quad \mathbf{T}-\mathbf{S R}^{-1} \mathbf{S}^{T}>0
$$

where the matrices $\mathbf{T}=\mathbf{T}^{T}, \mathbf{R}=\mathbf{R}^{T}$ and $\mathbf{S}$ are of appropriate dimension. Hence, the previous inequalities is equivalent to:

$$
\left[\begin{array}{rr}
\mathbf{T} & \mathbf{S} \\
\mathbf{S}^{T} & \mathbf{R}
\end{array}\right]>0
$$

\section{B. Conventional Camera Model}

Let the $3 \mathrm{D}$ point $\mathbf{p}$ represented in the camera frame with the coordinates $\mathbf{p}=\left(\begin{array}{lll}X Y Z & Y\end{array}\right)^{\top}$. Its projection onto the image plane is expressed by the homogeneous coordinates vector m as:

$$
\mathbf{m}=\left(\begin{array}{lll}
x & y & 1
\end{array}\right)^{\top}=\frac{1}{Z} \mathbf{p}
$$

The derivative of $\mathbf{p}$ is related to the spatial velocity of the camera $\mathbf{u}$ by the following equation:

$$
\dot{\mathbf{p}}=-v+\mathbf{p} \times \omega=\left(\begin{array}{ll}
-\mathbf{I}_{\mathbf{n}} & \left.[\mathbf{p}]_{\times}\right) \mathbf{u}
\end{array}\right.
$$

where $\mathbf{u}=\left(v^{\top} \omega^{\top}\right)^{\top}$ with $v=\left(v_{x} v_{y} v_{z}\right)^{\top}$ and $\omega=$ $\left(\omega_{x} \omega_{y} \omega_{z}\right)^{\top}$ are respectively, the instantaneous linear and angular velocity of the origin of the camera expressed in its local frame.

Let us define the measurable variables of the state vector as

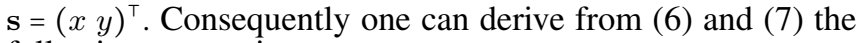
following expression:

$$
\dot{\mathbf{s}}=\left(\begin{array}{cccccc}
-\frac{1}{Z} & 0 & \frac{x}{Z} & x y & -\left(1+x^{2}\right) & y \\
0 & -\frac{1}{Z} & \frac{y}{Z} & \left(1+y^{2}\right) & -x y & -x
\end{array}\right) \mathbf{u}
$$

Applying dynamic extension allows to built a new system with less nonlinear variables in order to reduce the number of subsystems when considering the polytopic form.

We denote $\frac{1}{Z}$ and $\frac{v_{z}}{Z}$ as $\chi$ and $\eta$ respectively and we consider the state vector defined as $\mathbf{x}=\left(\mathbf{s}^{\top} \zeta^{\top}\right)^{\top}$ where $\zeta=(\chi \eta)^{\top}$ is the unmeasurable data to be estimated.

Remark 1. In the proposed work we consider the camera and the IMU are mounted very close by in a way that both their local frames axes are parallel. Therefore, we assume that both the proper acceleration and the angular velocity provided by the IMU expressed in its local frame are approximately the same measurements expressed in the camera local frame.

The accelerometer provides the measurement $\alpha_{s}$ expressed in the following equation [14]:

$$
\alpha_{s}=[\omega]_{\times} v+\dot{v}+g \mathbf{R}^{\top} \mathbf{e}_{z}
$$

which is the sum of the gravitational field and the linear acceleration of the sensor, where $\alpha_{s} \in \mathbb{R}^{3}$ represents the proper acceleration of the camera with respect to the inertial frame, expressed in the camera local frame, $\omega \in \mathbb{R}^{3}$ is the angular velocity measured by the gyroscope and expressed in the camera frame, $g \mathbf{R}^{\top} \mathbf{e}_{z}$ is the gravity measurement expressed in the local frame where $\mathbf{R}$ denotes the rotation matrix of the local frame with respect to the world frame and $g$ is the gravity constant expressed in the inertial frame and $\mathbf{e}_{z}$ is a unit vector collinear with the gravitational field. We introduce acceleration due to motion $\alpha_{a c c}=$ $\left(\alpha_{a c c_{x}} \alpha_{a c c_{y}} \alpha_{a c c_{z}}\right)^{\top}$, by subtracting the gravity offset as follows:

$$
\alpha_{a c c}=\alpha_{s}-g \mathbf{R}^{\top} e_{z}
$$

Remark 2. In order to compute the acceleration due to motion, an estimation of the attitude is necessary. Many works have addressed the attitude estimation, as in [12], where the attitude is estimated assuming a negligible linear accelerations compared to gravity. Also, in [13], a partial (tilt observation) and complete attitude estimators are presented. 
Then the equation (9) is equivalent to:

$$
\dot{v}=-[\omega]_{\times} v+\alpha_{a c c}
$$

The dynamics of $\chi$ and $\eta$ are deduced from (7) and (11) as follows:

$$
\begin{aligned}
\dot{\chi} & =\chi \eta+y \chi \omega_{x}-x \chi \omega_{y} \\
\dot{\eta} & =\alpha_{a c c_{z}} \chi-\omega_{y} v_{x} \chi+\omega_{x} v_{y} \chi+\eta^{2}-y \eta \omega_{x}+x \omega_{y} \eta
\end{aligned}
$$

The equations (8), (12) are arranged in the following form:

$$
\left\{\begin{aligned}
\dot{\mathbf{s}} & =\mathbf{f}_{m}(\mathbf{s}, \mathbf{u})+\boldsymbol{\Omega}(\mathbf{s}, \mathbf{u}) \zeta \\
\dot{\zeta} & =\mathbf{f}_{u}(\mathbf{s}, \zeta, \mathbf{u})
\end{aligned}\right.
$$

where $\boldsymbol{\Omega}^{\top}(\mathbf{s}, \mathbf{u}) \in \mathbb{R}^{2}, \mathbf{f}_{m}(\mathbf{s}, \mathbf{u}) \in \mathbb{R}^{2 \times 1}$, and $\mathbf{f}_{u}(\mathbf{s}, \chi, \mathbf{u}) \in \mathbb{R}^{2 \times 1}$ defined in (14) are sufficiently smooth w.r.t their arguments.

$$
\left\{\begin{aligned}
\mathbf{f}_{m}(\mathbf{s}, \mathbf{u}) & =\left(\begin{array}{ccc}
x y & -\left(1+x^{2}\right) & y \\
1+y^{2} & -x y & -x
\end{array}\right) \omega \\
\boldsymbol{\Omega}(\mathbf{s}, \mathbf{u}) & =\left(\begin{array}{ll}
-v_{x} & x \\
-v_{y} & y
\end{array}\right) \\
\mathbf{f}_{u}(\mathbf{s}, \chi, \mathbf{u}) & =\left(\begin{array}{c}
-\chi \eta-y \chi \omega_{x}+x \chi \omega_{y} \\
\alpha_{a c c_{z}} \chi-\omega_{y} v_{x} \chi+\omega_{x} v_{y} \chi+\eta^{2}- \\
y \eta \omega_{x}+x \omega_{y} \eta
\end{array}\right)
\end{aligned}\right.
$$

For readability sake, the time dependency is omitted and parameter dependency is represented as a subscript.

In the next section, the system in (13) will be described in a qLPV representation in order to design the observer.

\section{QUASI-LPV MODEL}

The objective of this work is to estimate the depth information $\chi$ and recover the linear velocity $v$ during the camera motion using a nonlinear unknown input observer. For this aim, the system (13) is written in a qLPV state space representation, described by:

$$
\left\{\begin{aligned}
\dot{\mathbf{x}} & =\mathbf{A}_{\mathbf{x}, \mathbf{u}} \mathbf{x}+\mathbf{B}_{\mathbf{y}} \omega+\mathbf{F}_{\mathbf{u}} \mathbf{d} \\
\mathbf{y} & =\mathbf{C}_{\mathbf{x}}
\end{aligned}\right.
$$

where $\mathbf{A}_{\mathbf{x}, \mathbf{u}} \in \mathbb{R}^{4 \times 4}, \mathbf{B}_{\mathbf{y}} \in \mathbb{R}^{4 \times 3}$, and $\mathbf{F}_{\mathbf{u}} \in \mathbb{R}^{4 \times 2}$. The following vectors $\mathbf{x} \in \mathbb{R}^{4}, \omega \in \mathbb{R}^{3}, \mathbf{d} \in \mathbb{R}^{2}$, and $\mathbf{y} \in \mathbb{R}^{2}$ are respectively the state vector, the angular velocity, the unknown input vector and the output vector, assumed to be bounded.

The matrices defined in the system above have the following form:

$$
\begin{aligned}
& \mathbf{A}_{\mathbf{x}, \mathbf{u}}=\left(\begin{array}{cccc}
0 & 0 & 0 & x \\
0 & 0 & 0 & y \\
0 & 0 & y \omega_{x}-x \omega_{y}+\eta & 0 \\
0 & 0 & \alpha_{a c c_{z}} & y \omega_{x}-x \omega_{y}+\eta
\end{array}\right) \\
& \mathbf{B}_{\mathbf{y}}=\left(\begin{array}{ccc}
x y & -\left(1+x^{2}\right) & y \\
1+y^{2} & -x y & -x \\
0 & 0 & 0 \\
0 & 0 & 0
\end{array}\right) \quad \mathbf{F}_{\mathbf{u}}=\left(\begin{array}{cc}
-1 & 0 \\
0 & -1 \\
0 & 0 \\
\omega_{y} & -\omega_{x}
\end{array}\right) \\
& \mathbf{d}=\left(\begin{array}{l}
\chi v_{x} \\
\chi v_{y}
\end{array}\right) \quad \mathbf{C}=\left(\begin{array}{cccc}
1 & 0 & 0 & 0 \\
0 & 1 & 0 & 0
\end{array}\right)
\end{aligned}
$$

We address in the next section the observer design following the conventional unknown input observer scheme for qLPV systems, considering the same framework demonstrated in
[18] where the Linear Parameter Varying (LPV) system representation is kept unchanged until establishing the LMI conditions in order to overcome this difficulty of imposing a structure for the gain matrices.

\section{OBSERVER DESIGN}

After transforming the system into qLPV form, this section deals with the observer design, in this context we adopt the following assumptions

Assumption 1: For a frozen values of the variables, we consider that [24]:

1) The state vector and the system inputs are considered bounded.

2) $\operatorname{rank}\left(\mathbf{C F}_{\mathbf{u}}\right)=\operatorname{rank}\left(\mathbf{F}_{\mathbf{u}}\right)$.

3) The pair $\left(\mathbf{T}_{\mathbf{u}} \mathbf{A}_{\mathbf{x}, \mathbf{u}}+\dot{\mathbf{T}}_{\mathbf{u}, \dot{\mathbf{u}}}, \mathbf{C}\right)$ is observable (or at least detectable). Where the matrix $\mathbf{T}_{\mathbf{u}}$ is computed as follows:

$$
\left\{\begin{array}{l}
\mathbf{E}_{\mathbf{u}}=\mathbf{F}_{\mathbf{u}}\left(\mathbf{C} \mathbf{F}_{\mathbf{u}}\right)^{-1} \\
\mathbf{T}_{\mathbf{u}}=\mathbf{I}-\mathbf{E}_{\mathbf{u}} \mathbf{C}
\end{array}\right.
$$

\section{A. Observer Structure}

Let us define the following observer:

$$
\left\{\begin{array}{l}
\dot{\mathbf{z}}=\mathbf{N}_{\hat{\mathbf{x}}, \mathbf{u}} \mathbf{z}+\mathbf{G}_{\mathbf{y}} \omega+\mathbf{L}_{\hat{\mathbf{x}}} \mathbf{y} \\
\hat{\mathbf{x}}=\mathbf{z}-\mathbf{E}_{\mathbf{u}} \mathbf{y}
\end{array}\right.
$$

where $\mathbf{z} \in \mathbb{R}^{4}$ is the state of the observer and $\hat{\mathbf{x}} \in \mathbb{R}^{4}$ the estimated state and the matrices $\mathbf{N}_{\hat{\mathbf{x}}}, \mathbf{G}_{\mathbf{y}}, \mathbf{L}_{\hat{\mathbf{x}}}$ and $\mathbf{E}_{\mathbf{u}}$ are gain matrices that guarantee the stability of the state estimation error.

The estimation error is expressed by the following form:

$$
\begin{aligned}
\mathbf{e} & =\mathbf{x}-\hat{\mathbf{x}} \\
& =\mathbf{T}_{\mathbf{u}} \mathbf{x}-\mathbf{z}
\end{aligned}
$$

where: $\quad \mathbf{T}_{\mathbf{u}}=\mathbf{I}+\mathbf{E}_{\mathbf{u}} \mathbf{C}$

Then the dynamics of the estimation error is given by:

$$
\begin{aligned}
\dot{\mathbf{e}}= & \mathbf{T}_{\mathbf{u}} \dot{\mathbf{x}}-\dot{\mathbf{z}} \\
= & \mathbf{N}_{\hat{\mathbf{x}}, \mathbf{u}} \mathbf{e}+\left(\mathbf{T}_{\mathbf{u}} \mathbf{A}_{\hat{\mathbf{x}}, \mathbf{u}}-\mathbf{K}_{\hat{\mathbf{x}}, \mathbf{u}} \mathbf{C}-\mathbf{N}_{\hat{\mathbf{x}}, \mathbf{u}}+\dot{\mathbf{T}}_{\mathbf{u}, \dot{\mathbf{u}}}\right) \mathbf{x}+ \\
& \mathbf{T}_{\mathbf{u}} \mathbf{F}_{\mathbf{u}} \mathbf{d}+\left(\mathbf{T}_{\mathbf{u}} \mathbf{B}_{\mathbf{y}}-\mathbf{G}_{\mathbf{y}}\right) \omega+\mathbf{T}_{\mathbf{u}} \delta_{\varrho, \mathbf{u}}
\end{aligned}
$$

with $\delta_{\varrho, \mathbf{u}}=\left(\mathbf{A}_{\mathbf{x}, \mathbf{u}}-\mathbf{A}_{\hat{\mathbf{x}}, \mathbf{u}}\right) \mathrm{X}, \varrho=(\hat{\mathbf{x}}, \mathbf{x})$ and $\mathbf{K}_{\hat{\mathbf{x}}, \mathbf{u}}=\mathbf{N}_{\hat{\mathbf{x}}, \mathbf{u}} \mathbf{F}_{\mathbf{u}}-$ $\mathbf{L}_{\hat{\mathbf{x}}}$.

Under the following conditions:

$$
\begin{aligned}
& \text { 1) } \mathbf{T}_{\mathbf{u}} \mathbf{A}_{\hat{\mathbf{x}}, \mathbf{u}}-\mathbf{K}_{\hat{\mathbf{x}}, \mathbf{u}} \mathbf{C}-\mathbf{N}_{\hat{\mathbf{x}}, \mathbf{u}}+\dot{\mathbf{T}}_{\mathbf{u}, \dot{\mathbf{u}}}=0 \\
& \text { 2) } \mathbf{T}_{\mathbf{u}} \mathbf{B}_{\mathbf{y}}-\mathbf{G}_{\mathbf{y}}=0 \\
& \text { 3) } \mathbf{T}_{\mathbf{u}} \mathbf{F}_{\mathbf{u}}=0
\end{aligned}
$$

The dynamics of the estimation error becomes:

$$
\dot{\mathbf{e}}=\mathbf{N}_{\hat{\mathbf{x}}, \mathbf{u}} \mathbf{e}+\mathbf{T}_{\mathbf{u}} \delta_{\varrho}, \mathbf{u}
$$

Hence the following equations can be obtained:

$$
\begin{aligned}
\mathbf{N}_{\hat{\mathbf{x}}, \mathbf{u}} & =\boldsymbol{\Gamma}_{\hat{\mathbf{x}}, \mathbf{u}}-\mathbf{K}_{\hat{\mathbf{x}}, \mathbf{u}} \mathbf{C} \\
\mathbf{E}_{\mathbf{u}} & =\mathbf{F}_{\mathbf{u}}\left(\mathbf{C} \mathbf{F}_{\mathbf{u}}\right)^{-1} \\
\mathbf{G}_{\mathbf{y}} & =\mathbf{T}_{\mathbf{u}} \mathbf{B}_{\mathbf{y}}
\end{aligned}
$$

where: $\boldsymbol{\Gamma}_{\hat{\mathbf{x}}, \mathbf{u}}=\mathbf{N}_{\hat{\mathbf{x}}, \mathbf{u}}=\mathbf{T}_{\mathbf{u}} \mathbf{A}_{\hat{\mathbf{x}}, \mathbf{u}}+\dot{\mathbf{T}}_{\mathbf{u}, \dot{\mathbf{u}}}$

$$
\mathbf{E}_{\mathbf{u}}=\left(\begin{array}{cc}
-1 & 0 \\
0 & -1 \\
0 & 0 \\
-\omega_{y} & \omega_{x}
\end{array}\right) \quad \mathbf{T}_{\mathbf{u}}=\left(\begin{array}{cccc}
0 & 0 & 0 & 0 \\
0 & 0 & 0 & 0 \\
0 & 0 & 1 & 0 \\
-\omega_{y} & \omega_{x} & 0 & 1
\end{array}\right)
$$




$$
\dot{\mathbf{T}}_{\mathbf{u}, \dot{\mathbf{u}}}=\left(\begin{array}{cccc}
0 & 0 & 0 & 0 \\
0 & 0 & 0 & 0 \\
0 & 0 & 0 & 0 \\
-\dot{\omega}_{y} & \dot{\omega}_{x} & 0 & 0
\end{array}\right)
$$

Remark 3. Tools aiming to measure the angular acceleration are not yet popular. But among the works that addressed this problem, one can cite [19] where sensors, methods, and algorithms available for the measurement of angular acceleration are given. Also the time derivative of the angular velocity can be obtained using advanced differentiator designs (see [20] and references therein).

\section{B. Observer Convergence Study}

The error dynamic system (20) can be represented as a Takagi-Sugeno form as follows:

$$
\begin{aligned}
\dot{\mathbf{e}} & =\mathbf{N}_{\hat{\mathbf{x}}, \mathbf{u}} \mathbf{e}+\mathbf{T}_{\mathbf{u}} \delta_{\varrho, \mathbf{u}} . \\
& =\left(\boldsymbol{\Gamma}_{\hat{\mathbf{x}}, \mathbf{u}}-\mathbf{K}_{\hat{\mathbf{x}}, \mathbf{u}} \mathbf{C}\right) \mathbf{e}+\mathbf{T}_{\mathbf{u}} \delta_{\varrho, \mathbf{u}} \\
& =\sum_{i=1}^{r} \mu_{i}(\hat{\mathbf{x}}, \mathbf{x})\left(\left(\boldsymbol{\Gamma}_{i}-\mathbf{K}_{i} \mathbf{C}\right) \mathbf{e}+\mathbf{T}_{i} \delta_{\varrho, \mathbf{u}}\right)
\end{aligned}
$$

where $\boldsymbol{\Gamma}_{\mathbf{i}} \in \mathbb{R}^{4 \times 4}, \mathbf{T}_{\mathbf{i}} \in \mathbb{R}^{4 \times 4}$ and $\mu_{i}(\hat{\mathbf{x}}, \mathbf{x}), i=1, \ldots, 2^{n}$ are the weighing functions satisfying the convex sum property stated in (25). The T-S model allows to have an exact representation of the initial nonlinear system described as multiple linear sub-models, so that for $n$ nonlinearities, we obtain $r=2^{n}$ sub-model [17]. In our case, we have 7 nonlinearities, which makes $r=2^{7}$ sub-model.

$$
\left\{\begin{array}{r}
0 \leqslant \mu_{i}(\varrho, \mathbf{u}) \leqslant 1 \\
\sum_{i=1}^{r} \mu_{i}(\varrho, \mathbf{u})=1
\end{array}\right.
$$

Theorem 1: The error dynamics (24) is ISS with respect to $\delta_{\varrho, \mathbf{u}}$ with minimized ISS gain $\phi_{2}$ :

$$
\|(\mathbf{x}(t))\|_{2}<\phi_{1}\|(\mathbf{x}(0))\|_{2} e^{-\frac{\sigma}{2} t}+\phi_{2} \|\left(\delta_{\varrho}, \mathbf{u} \|_{\infty}\right.
$$

If it exists a positive definite matrix $\mathbf{F}$ and a symmetric positive definite matrix $\mathbf{P}$ with the minimal and maximal eigenvalues denoted respectively by $\mathcal{X}_{1}$ and $\mathcal{X}_{2}$, such that the following LMIs conditions for a given $\sigma$ and $\mathcal{X}_{1}$ hold $\forall i=1, \ldots, r$ :

$$
\begin{gathered}
\min _{\mathbf{P}, \mathbf{F}} \gamma \\
\mathbf{F} \leq \gamma \mathbf{I} \\
\left(\begin{array}{cc}
\boldsymbol{\Gamma}_{i}^{\top} \mathbf{P}+\mathbf{C}^{\top} \mathbf{W}_{i}^{\top}+\mathbf{P} \mathbf{\Gamma}_{i}+\mathbf{W}_{i} \mathbf{C}+\sigma \mathbf{P} & \mathbf{P} \mathbf{T}_{i} \\
\mathbf{T}_{i}^{\top} \mathbf{P} & -\mathbf{F}
\end{array}\right)<0
\end{gathered}
$$

with: $\mathbf{W}_{i}=\mathbf{P K}_{i}, \phi_{1}=\sqrt{\frac{\mathcal{X}_{2}}{\mathcal{X}_{1}}}, \phi_{2}=\sqrt{\frac{\gamma}{\sigma \mathcal{X}_{1}}}$, and $\mathcal{X}_{1} \mathbf{I} \leqslant \mathbf{P} \leqslant \mathcal{X}_{2} \mathbf{I}$

Proof: Considering the quadratic Lyapunov function given by:

$$
V(t)=\mathbf{e}(t)^{\top} \mathbf{P e}(t) \quad \mathbf{P}=\mathbf{P}^{\top}>0
$$

Since Lyapunov function is positive definite, it can be upper and lower bounded as follows:

$$
\mathcal{X}_{1}\|(\mathbf{e}(0))\|^{2} \leqslant V(t) \leqslant \mathcal{X}_{2}\|(\mathbf{e}(0))\|^{2}
$$

where $\mathcal{X}_{1}$ and $\mathcal{X}_{2}$ are the minimum and maximum eigenvalues of the matrix $\mathbf{P}$.

For shortness we write $\delta(\hat{\mathbf{x}}, \mathbf{x})$ as $\delta$. Taking the time derivative of Lyapunov function (28), one obtains:

$$
\dot{V}(t)=\mathbf{e}^{\top}\left(\mathbf{N}_{\hat{\mathbf{x}}, \mathbf{u}}^{\top} \mathbf{P}+\mathbf{P} \mathbf{N}_{\hat{\mathbf{x}}, \mathbf{u}}\right) \mathbf{e}+2 \mathbf{e} \mathbf{P} \mathbf{T}_{\mathbf{u}} \delta
$$

Taking into consideration lemma 1, yields:

$$
\begin{aligned}
\dot{V}(t)= & \mathbf{e}^{\top}\left(\mathbf{N}_{\hat{\mathbf{x}}, \mathbf{u}}^{\top} \mathbf{P}+\mathbf{P} \mathbf{N}_{\hat{\mathbf{x}}, \mathbf{u}}+\mathbf{P} \mathbf{T}_{\mathbf{u}} \mathbf{F}^{-\mathbf{1}} \mathbf{T}_{\mathbf{u}}^{\top} \mathbf{P}+\sigma \mathbf{P}\right) \mathbf{e}+ \\
& \delta^{\top} \mathbf{F} \delta-\sigma \mathbf{e}^{\top} \mathbf{P} \mathbf{e}
\end{aligned}
$$

where $\sigma$ is a positive real constant.

We accept that:

$$
\mathbf{e}^{\top}\left(\mathbf{N}_{\hat{\mathbf{x}}, \mathbf{u}}^{\top} \mathbf{P}+\mathbf{P} \mathbf{N}_{\hat{\mathbf{x}}, \mathbf{u}}+\mathbf{P} \mathbf{T}_{\mathbf{u}} \mathbf{F}^{-\mathbf{1}} \mathbf{T}_{\mathbf{u}}^{\top} \mathbf{P}+\sigma \mathbf{P}\right) \mathbf{e} \leq 0
$$

Applying lemma 2, and substituting $\mathbf{N}_{\hat{\mathbf{x}}, \mathbf{u}}$ in (21), the inequality (32) is equivalent to:

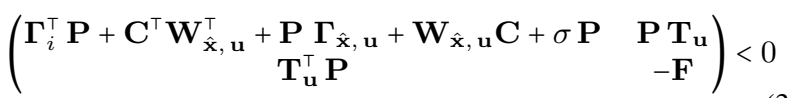

where: $\mathbf{W}_{\hat{\mathbf{x}}, \mathbf{u}}=\mathbf{P} \mathbf{K}_{\hat{\mathbf{x}}, \mathbf{u}}$

Respecting (33), implies the following new bounding of Lyapunov function derivative:

$$
V(t)<-\sigma \mathbf{e}^{\top} \mathbf{P} \mathbf{e}+\delta^{\top} \mathbf{F} \delta
$$

Integrating the previous inequality (34), yields:

$$
\begin{aligned}
V(t) & <V(0) e^{-\sigma t}+\mathbf{F} \int_{0}^{t} e^{-\sigma(t-s) t}\|\delta(s)\|^{2} d s \\
& \leq V(0) e^{-\sigma t}+\frac{\gamma}{\sigma}\|\delta(s)\|_{\infty}^{2}
\end{aligned}
$$

Taking into account the bounds of Lyapunov function in (29), we obtain:

$$
\|(\mathbf{e}(t))\|^{2}<\frac{\mathcal{X}_{2}}{\mathcal{X}_{1}}\|(\mathbf{e}(0))\|^{2} e^{-\sigma t}+\frac{\mathbf{F}}{\sigma \mathcal{X}_{1}}\|(\delta(s))\|_{\infty}^{2}
$$

Also, if we consider the inequality (27b), we get:

$$
\|(\mathbf{e}(t))\|^{2}<\frac{\mathcal{X}_{2}}{\mathcal{X}_{1}}\|(\mathbf{e}(0))\|^{2} e^{-\sigma t}+\frac{\gamma}{\sigma \mathcal{X}_{1}}\|(\delta(s))\|_{\infty}^{2}
$$

which yields the following:

$$
\|(\mathbf{e}(t))\|<\sqrt{\frac{\mathcal{X}_{2}}{\mathcal{X}_{1}}}\|(\mathbf{e}(0))\| e^{-\frac{\sigma}{2} t}+\sqrt{\frac{\gamma}{\sigma \mathcal{X}_{1}}}\|(\delta)\|_{\infty}
$$

This inequality verifies the ISS property of the system (20) with respect to the perturbation $\delta$ with minimal ISS gain ensured by the condition (27a) and explicitly expressed by $\phi_{2}=\sqrt{\frac{\gamma}{\sigma \mathcal{X}_{1}}}$.

Minimizing the variable $\gamma$ will attenuate the effect of the disturbance $\delta$ and ensures a minimal bound of the estimation error.

\section{Unknown Input Reconstruction}

Now, we are interested in reconstructing the linear camera velocity. For this aim, we compute the time derivative of the equation defining the measurements output in (15), accordingly:

$$
\dot{\hat{\mathbf{y}}}=\mathbf{C}\left(\mathbf{A}_{\hat{\mathbf{x}}, \mathbf{u}} \hat{\mathbf{x}}+\mathbf{B}_{\mathbf{y}} \omega+\mathbf{F}_{\mathbf{u}} \hat{\mathbf{d}}\right)
$$

Then the disturbance estimate takes the following form:

$$
\left.\hat{\mathbf{d}}=\left(\mathbf{C} \mathbf{F}_{\mathbf{u}}\right)^{-1}\left(\dot{\hat{\mathbf{y}}}-\mathbf{C}\left(\mathbf{A}_{\hat{\mathbf{x}}, \mathbf{u}} \hat{\mathbf{x}}+\mathbf{B}_{\mathbf{y}} \omega\right)\right)\right)
$$

Thus, the camera linear velocity $v_{x}$ and $v_{y}$ are deduced using the following equation :

$$
\hat{\mathbf{d}}=\left(\begin{array}{ll}
\hat{\chi} & \hat{v}_{x} \\
\hat{\chi} & \hat{v}_{y}
\end{array}\right) \quad \Longrightarrow\left(\begin{array}{l}
\hat{v}_{x} \\
\hat{v}_{y}
\end{array}\right)=\hat{\mathbf{d}}(t) \hat{\chi}^{-1}
$$


And finally the linear velocity w.r.t the $z$ axis of the camera $\hat{v}_{z}$ is obtained directly from the estimated states $\hat{\eta}$ and $\hat{\chi}$, thus:

$$
\hat{v}_{z}=\hat{\eta} \hat{\chi}^{-1}
$$

A recap of the observer design procedure is given in the following steps:

1) Verify the observability condition of the pair $\left(\mathbf{T}_{\mathbf{u}} \mathbf{A}_{\mathbf{x}, \mathbf{u}}+\dot{\mathbf{T}}_{\mathbf{u}, \dot{\mathbf{u}}}, \mathbf{C}\right)$ and the rank condition $\operatorname{rank}\left(\mathbf{C F}_{\mathbf{u}}\right)=\operatorname{rank}\left(\mathbf{F}_{\mathbf{u}}\right)$.

2) Compute the matrices $\mathbf{E}_{\mathbf{u}}, \mathbf{T}_{\hat{\mathbf{x}}, \mathbf{u}}$ directly since they are all dependent on measured states, as well as the matrix $\mathbf{G}_{\mathbf{y}}$ given by the equations (16) and (23) respectively.

3) Solve the LMIs constraints (27) for a given positive scaler $\sigma$ and $\mathcal{X}_{1}$, and calculate the gains $\mathbf{W}_{\hat{\mathrm{x}}}$ and $\Gamma_{\hat{\mathrm{x}}}$ as follows:

$$
\left\{\begin{aligned}
\mathbf{W}_{\hat{\mathbf{x}}, \mathbf{u}} & =\sum_{i=1}^{r} \mu_{i}(\hat{\mathbf{x}}, \mathbf{x}) \mathbf{W}_{i} \\
\boldsymbol{\Gamma}_{\hat{\mathbf{x}}, \mathbf{u}} & =\sum_{i=1}^{r} \mu_{i}(\hat{\mathbf{x}}, \mathbf{x}) \boldsymbol{\Gamma}_{i}
\end{aligned}\right.
$$

4) Deduce the observer gain matrices $\mathbf{N}_{\hat{\mathbf{x}}, \mathbf{u}}$ and $\mathbf{L}_{\hat{\mathbf{x}}, \mathbf{u}}$, using:

$$
\left\{\begin{array}{l}
\mathbf{K}_{\hat{\mathbf{x}}, \mathbf{u}}=\mathbf{P}^{-1} \mathbf{W}_{\hat{\mathbf{x}}} \\
\mathbf{N}_{\hat{\mathbf{x}}, \mathbf{u}}=\mathbf{T}_{\mathbf{u}} \mathbf{A}_{\hat{\mathbf{x}}}+\dot{\mathbf{T}}_{\mathbf{u}, \dot{\mathbf{u}}}-\mathbf{K}_{\hat{\mathbf{x}}} \mathbf{C}
\end{array}\right.
$$

5) Finally, use the equations (42) and (41) to obtain the estimated camera linear velocity.

In the next section simulation results are reported to demonstrate the proposed approach.

\section{SIMULATION RESULTS}

This section reports the simulation results in order to illustrate the applicability of the proposed approach. For this aim, synthetic data set of images is generated at a rate of $33 \mathrm{fps}$ using a known camera angular and linear velocities $\omega$ and $v$ given below in (45). The orientation matrix $\mathbf{R}$ of the sensor with respect to the world frame is obtained by integration, and the accelerometer signal $\alpha_{s}$ is computed using equation (9).

For $\mathcal{X}_{1}=10^{-12}$ and $\sigma=10^{-2}$, the result obtained from solving the LMIs in (27) using Yalmip toolbox in Matlab are given by:

$$
\begin{gathered}
\gamma=9.5492 \cdot 10^{-13} \\
\mathbf{P}=\left(\begin{array}{cccc}
0.0034 & -2.0580 \cdot 10^{-8} & 0.0 & 0.0 \\
-2.0580 \cdot 10^{-8} & 0.0034 & 0.0 & 0.0 \\
0.0 & 0.0 & 9.8857 \cdot 10^{-13} & 0.0 \\
0.0 & 0.0 & 0.0 & 1.6109 \cdot 10^{-13}
\end{array}\right)
\end{gathered}
$$

We assume that the camera is calibrated and it is initially at a position of 1 meter $(Z=1)$ from the tracked point, chosen to be the center of the image.

The camera linear and angular velocities are given by:

$$
\begin{aligned}
& v_{x}=0.05 \sin \left(\frac{\pi}{5} t\right), v_{y}=\frac{1}{(t+5)}, v_{z}=-0.3 e^{-0.02 t}-0.1 \sin \left(\frac{\pi}{6} t\right) \\
& \omega_{x}=0.05 \cos \left(\frac{\pi}{4} t\right), \omega_{y}=-0.06 \cos \left(\frac{\pi}{2} t\right),, \omega_{z}=0
\end{aligned}
$$

The observer initial conditions are: $\mathbf{z}=\left(\begin{array}{ll}1,2, & 4,-1.6\end{array}\right)^{\top}$. a) Case 1: considering known measurements: Simulations in Fig. 1 and Fig. 2 are given considering known measurements. These figures show that the variables $\chi, \eta$ and the linear velocity are successfully estimated.

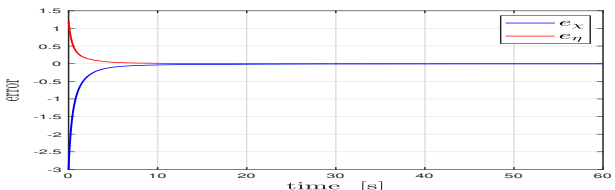

Fig. 1: The estimation error $e_{\chi}=\chi-\hat{\chi}$ in solid blue line and the estimation error $e_{\eta}=\eta-\hat{\eta}$ in solid red line

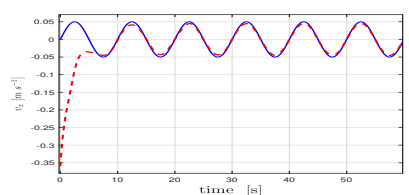

(a)

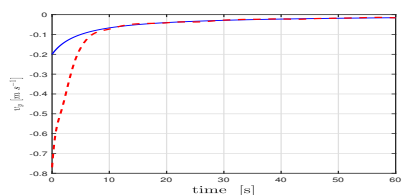

(b)

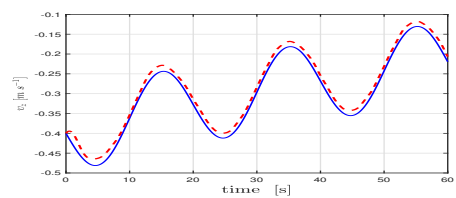

(c)

Fig. 2: Real (solid bleu line) and estimated (dashed red line) linear velocities: (a) $v_{x}$, (b) $v_{y}$ and (c) $v_{z}$.

b) Case 2: taking into account measurements uncertainties: Now, In order to reassess the robustness of the observer to measurements uncertainties, the values shown in table I, quantifies the error performance based on the Root Mean Squared Error (RMSE) for different scenarios. In each scenario a random number of pixels within the considered interval is added to the exact position of the tracked pixel in each iteration.

The RMSE formula for a variable $\kappa$ is given as:

$$
R M S E=\sqrt{\frac{1}{n} \Sigma_{i=1}^{n}(\kappa-\hat{\kappa})_{i}^{2}}
$$

Where: $n$ is the number of $\kappa$ error samples.

TABLE I: Comparison of the estimation error for different scenarios based on RMSE

\begin{tabular}{|c|ccccc|}
\cline { 2 - 6 } \multicolumn{1}{c|}{} & $\chi$ & $\eta$ & $v_{x}$ & $v_{y}$ & $v_{z}$ \\
\hline 0 & 0.2350 & 0.0917 & 0.0516 & 0.0848 & 0.0139 \\
\hline$[-2,2]$ & 0.2319 & 0.0937 & 0.5483 & 0.9726 & 0.0196 \\
\hline$[-4,4]$ & 0.2319 & 0.0937 & 1.1204 & 1.9462 & 0.0198 \\
\hline$[-8,8]$ & 0.2321 & 0.0938 & 2.2697 & 3.9192 & 0.0199 \\
\hline
\end{tabular}

The table I indicates that the RMSE values of the estimation error of the unmeasured states $(\chi$ and $\eta)$ depict a small variations. Same observation for the RMSE values of estimated velocity $v_{z}$, Hence, we can say that the observer shows 
a robustness to measurements uncertainties and it ensures an accurate state estimation. That is basically due to the optimization of the bound of the estimation error. In this case the uncertainties of the measurements will be added to the perturbation vector $\delta_{\varrho, \mathbf{u}}$ during the design procedure. Then, the same previous study is performed. Consequently, the estimation error will have another optimized bound different than the one obtained when considering known measurements. It should be noted that the ISS property is more effective comparing to other stability properties like $L_{2}$ stability or the Input to State practical Stability (ISpS) [25]. On the other hand, one can notice that the RMSE values for the reconstructed velocities $v_{x}$ and $v_{y}$ are increasing, meaning that they exhibit a noisy convergence. That is essentially resulting from the amplification of the noise due to the differentiation of the measured state in equation (41) to reconstruct both velocities. However, we could have had better performance using data filtering techniques.

\section{CONCLUSION}

In this paper, the design of an unknown input observer for qLPV systems with unmeasured premise variables is proposed, to estimate the depth information and the linear camera velocity. The considered system is obtained by a dynamic extension of a vision camera system. Necessary and sufficient conditions are provided to ensure the existence of the observer. The convergence of the estimation error is investigated based on the ISS property and Lyapunov analysis. The observer gains are computed by solving LMI constrains. Finally, simulations are carried out using synthetic data to validate the proposed observer. The estimation performances are quantified with the RMSE criteria and tested for different scenarios.

The presented approach gives an interesting solution for linear velocity estimation which is very hard to measure, using only an Inertial Measurement Unit and a low cost camera. Also our approach shows a good performance in estimating of the depth information without strong assumptions comparing to other existing work which requires a known geometry of the observed object or full knowledge of the camera velocity.

\section{REFERENCES}

[1] Ström, J. (2001, March). Structure from motion of planar surfaces (analysis of an EKF based approach). In Proceedings of Symposium on Image Analysis, Swedish Society for Automated Image Analysis (pp. 13-16).

[2] Civera, J., Grasa, O. G., Davison, A. J., \& Montiel, J. M. M. (2010). 1-Point RANSAC for extended Kalman filtering: Application to realtime structure from motion and visual odometry. Journal of field robotics, 27(5), 609-631.

[3] Civera, J., Davison, A. J., \& Montiel, J. M. M. (2011). Structure from motion using the extended Kalman filter (Vol. 75). Springer Science \& Business Media.

[4] Van Wyk, B. M., Muller, N. L., \& Hunter, K. M. (2007). Improving UKF performance.

[5] Omari, S., \& Ducard, G. (2013, July). Metric visual-inertial navigation system using single optical flow feature. In 2013 European Control Conference (ECC) (pp. 1310-1316). IEEE.
[6] De Luca, A., Oriolo, G., \& Robuffo Giordano, P. (2008). Feature depth observation for image-based visual servoing: Theory and experiments. The International Journal of Robotics Research, 27(10), 1093-1116.

[7] Spica, R., \& Giordano, P. R. (2013, December). A framework for active estimation: Application to structure from motion. In 52nd IEEE conference on decision and control (pp. 7647-7653). IEEE.

[8] Mateus, A., Tahri, O., \& Miraldo, P. (2018, October). Active structurefrom-motion for 3d straight lines. In 2018 IEEE/RSJ International Conference on Intelligent Robots and Systems (IROS) (pp. 58195825). IEEE.

[9] Spica, R., Giordano, P. R., \& Chaumette, F. (2014). Active structure from motion: Application to point, sphere, and cylinder. IEEE Transactions on Robotics, 30(6), 1499-1513.

[10] Tahri, O., Giordano, P. R., \& Mezouar, Y. (2015, September). Rotation free active vision. In 2015 IEEE/RSJ International Conference on Intelligent Robots and Systems (IROS) (pp. 3086-3091). IEEE.

[11] Tahri, O., Boutat, D., \& Mezouar, Y. (2017). Brunovsky's linear form of incremental structure from motion. IEEE Transactions on Robotics, 33(6), 1491-1499.

[12] Mahony, R., Hamel, T., \& Pflimlin, J. M. (2008). Nonlinear complementary filters on the special orthogonal group. IEEE Transactions on automatic control, 53(5), 1203-1218.

[13] Benallegue, M., Benallegue, A., Cisneros, R., \& Chitour, Y. (2020). Velocity-Aided IMU-Based Attitude Estimation. arXiv preprint arXiv:2002.10205.

[14] Shala, U., \& Rodriguez, A. (2011). Indoor positioning using sensorfusion in android devices.

[15] Chhaya, F., Reddy, D., Upadhyay, S., Chari, V., Zia, M. Z., \& Krishna, K. M. (2016, May). Monocular reconstruction of vehicles: Combining slam with shape priors. In 2016 IEEE International Conference on Robotics and Automation (ICRA) (pp. 5758-5765). IEEE.

[16] Robenack, K., \& Palis, S. (2018, October). Nonlinear control of flat systems using a non-flat output with dynamic extension. In 2018 22nd International Conference on System Theory, Control and Computing (ICSTCC) (pp. 273-278). IEEE.

[17] Zhao, D., Lam, H. K., Li, Y., Ding, S. X., \& Liu, S. (2020). A Novel Approach to State and Unknown Input Estimation for Takagi-Sugeno Fuzzy Models With Applications to Fault Detection. IEEE Transactions on Circuits and Systems I: Regular Papers, 67(6), 2053-2063.

[18] Ichalal, D., \& Mammar, S. (2015). On unknown input observers for LPV systems. IEEE Transactions on Industrial Electronics, 62(9), 5870-5880.

[19] Ovaska, S. J., \& Valiviita, S. (1998, May). Angular acceleration measurement: A review. In IMTC/98 Conference Proceedings. IEEE Instrumentation and Measurement Technology Conference. Where Instrumentation is Going (Cat. No. 98CH36222) (Vol. 2, pp. 875-880). IEEE.

[20] Hongwei, W., \& Heping, W. (2015). A comparison study of advanced tracking differentiator design techniques. Procedia Engineering, 99, 1005-1013.

[21] Spica, R., Giordano, P. R., \& Chaumette, F. (2015, May). Plane estimation by active vision from point features and image moments. In 2015 IEEE International Conference on Robotics and Automation (ICRA) (pp. 6003-6010). IEEE.

[22] Hua, M. D., Manerikar, N., Hamel, T., \& Samson, C. (2018, May). Attitude, linear velocity and depth estimation of a camera observing a planar target using continuous homography and inertial data. In 2018 IEEE International Conference on Robotics and Automation (ICRA) (pp. 1429-1435). IEEE.

[23] Dani, A. P., Fischer, N. R., \& Dixon, W. E. (2011). Single camera structure and motion. IEEE Transactions on Automatic Control, 57(1), 238-243.

[24] Shamma, J. S. (2012). An overview of LPV systems. Control of linear parameter varying systems with applications, 3-26.

[25] Jiang, Z. P., Teel, A. R., \& Praly, L. (1994). Small-gain theorem for ISS systems and applications. Mathematics of Control, Signals and Systems, 7(2), 95-120. 